\title{
Elizabethkingia meningosepticum: an emerging nosocomial pathogen in a critical patient with septicaemia
}

\author{
Vittorio Panetta, ${ }^{1}$ Rita Greco, ${ }^{1}$ Sandra Costanzo,${ }^{2}$ Andrea Morello, ${ }^{2}$ Angelo Costanzo, ${ }^{1}$ Giuseppe Canzano ${ }^{1}$ \\ ${ }^{1}$ Microbiology Unit, Clinical Pathology Complex Operative Unit, Sant'Anna e San Sebastiano Hospital, Caserta; \\ ${ }^{2}$ Clinical Pathology, Second University of Naples, Italy
}

\begin{abstract}
Summary
We describe a case of sepsis due to the non-fermenter Gram-negative bacillus Elizabethkingia meningosepticum in a patient (M, 23 y.o.) hospitalized at the Intensive Care and Critical Care Medicine suffering of a severe neurological disease and acute surgical abdomen, caused by suppurative peritonitis secondary to PEG removal. Two sets of blood cultures were collected at the time of persistent fever. The culture on chocolate and blood agar showed the presence of colonies with characteristic grey-shiny appearance and 1-2 $\mathrm{mm}$ in diameter. No growth was instead evident on Mac-Conkey agar. The Gram stain was performed, but the final identification of the isolate as E. meningosepticum was obtained by Vitek2 System, on the basis of growth characteristics, biochemical reaction results, and the peculiar antibiotic susceptibility profile. The identification was also confirmed by Vitek MS MALDI-TOF mass spectrometry instrument (bioMérieux, Marcy-l'Étoile, France). Results of susceptibility testing highlighted the resistance
\end{abstract}

\footnotetext{
Correspondence: Vittorio Panetta, UOSD of Microbiology, UOC Clinical Pathology, AORN "S. Anna e San Sebastiano", Caserta, Italy. Tel.: +39.0823.232139/2523.

E-mail: vittorio.panetta@aorncaserta.it
}

Key words: Elizabethkingia meningosepticum; Septicaemia; Multi-drug resistant; nosocomial infection; Bloodstream infections.

Contributions: the authors contributed equally.

Conflict of interest: the authors declare no potential conflict of interest.

Received for publication: 6 March 2015 .

Revision received: 19 Janaury 2017.

Accepted for publication: 19 January 2017.

(C) Copyright V. Panetta et al., 2017

Licensee PAGEPress, Italy

Microbiologia Medica 2017; 32:5009

doi:10.4081/mm.2017.5009

This article is distributed under the terms of the Creative Commons Attribution Noncommercial License (by-nc 4.0) which permits any noncommercial use, distribution, and reproduction in any medium, provided the original author(s) and source are credited. to most antibiotics useful against Gram-negative bacteria and a paradoxical susceptibility to those targeting mainly Gram-positives. The vancomycin and ciprofloxacin combined therapy improved the patient's conditions, with a complete resolution of the E. meningosepticum bacteraemia. Unfortunately, due to the onset of important clinical and surgical complications, the patient died as a result of a septic shock caused by a MDR Acinetobacter baummannii. The present work underline the importance of correct species identification in the case of E. meningosepticum, a rare bacterium whose unique antimicrobial susceptibility pattern, require an ad hoc therapeutic approach.

\section{Introduction}

Elizabethkingia meningosepticum is a non-fermenter Gram-negative bacillus isolated for the first time in 1959 by the American bacteriologist Elizabeth King, who studied nonclassified bacilli related to childhood meningitis. The bacterium, initially classified as Flavobacterium meningosepticum, was re-classified as Cryseobacterium meningosepticum in 1994 and subsequently, after phylogenetic studies, definitely categorized as E. meningosepticum in $2005(3,9,8)$.

Widespread in nature (fresh water, salt water, or soil), is an aerobic, non-fermenter, non-spore-forming and non-mobile, saprophiticus ubiquitous bacillus. Unlike many other non-fermenter bacteria (Flavobacterium and Cryseobacterium), E. meningosepticum grows well at $37^{\circ} \mathrm{C}$ on blood agar and chocolate agar $(4,7)$. The colonies appear very pale yellow or more often not pigmented (dissimilarly to all Chryseobacterium species, that produce a not diffusible yellow-orange pigment); their growth is not easily appreciable after 24 hours of incubation (1). The grey colour of the colonies on blood agar is due to protease and gelatinase production. They grow poorly on MacConkey agar and are unable to grow on colistin-nalidixic acid (CNA) agar due to the susceptibility to quinolones such as nalidixic acid $(6,10,14)$.

E. meningosepticum has a characteristic resistance to most antibiotics useful against Gram-negative bacteria (aminoglycosides, beta-lactams, cloramphenicols, but it is susceptible to vancomycin, ciprofloxacin, levofloxacin, etc.. Due to the natural resistance to multiple antimicrobials 
it can colonize different hospital environments and medical equipment (such as incubation tubes, saline solution, syringes, medical devices, incubators, etc.) as an opportunistic nosocomial pathogen $(6,14)$ and can generate disease in favouring hospital and individual conditions. Most cases of the disease concern neonatal meningitis with a degree of mortality of $57 \%(5,12,15,13)$, although it may also represent a rare cause of pneumonia, endocarditis, postsurgical bacteraemia, cellulite and abdominal infections in immunosuppressed patients $(1-3,14)$. Therefore, immunosuppression, particular treatment and/or invasive surgical procedures, long-term care, use of a central venous catheter and prolonged and untargeted antibiotic prophylaxis are considered among the main risk factors $(12,16)$.

\section{Case report}

Patient A.A. (male, 23 years-old), suffering from mental retardation and paraplegic marked from birth with neuromuscular involvement characterized by hypotonia, muscular hypoplasia and cognitive impairment, was admitted to our Emergency for PEG's dislodgment, due to an acute abdomen, and needing an urgent surgical treatment for the PEG removal. Then, the stabilized patient was transferred to the Department of Intensive Care and Critical Care Medicine. The computed tomography scan showed the presence of fluid in the abdomen in perigastric seat, suprahepatic, and to the anterior abdominal wall, drains were therefore applied under the liver and in the pelvic cavity.

Laboratory tests reveal a WBC count of $23.7 \times 10^{3} / \mu \mathrm{L}$ with neutrophil (85\%), 12\% of lymphocytes, anaemia $\left(3.3 \times 10^{6} / \mu \mathrm{L}\right.$ RBC and $\left.8.5 \mathrm{mg} / \mathrm{dL}\right)$ and thrombocytopenia $\left(51 \times 10^{3} / \mu \mathrm{L}\right)$, urea $59 \mathrm{mg} / \mathrm{dL}$, creatinine $0.80 \mathrm{mg} / \mathrm{dL}$, and procalcitonin $5.36 \mathrm{ng} / \mathrm{mL}$. These clinical-instrumental data and persistent fever $\left(38-39^{\circ} \mathrm{C}\right)$, from the first day of hospitalization in intensive care, laid for a suspected sepsis caused by suppurative peritonitis. Therefore, 2 sets of blood cultures were performed (1st set on the day of admission and 2nd set at a distance of six days), a bacterial culture of peritoneal drainage fluid and an endotoxin dosage (EAA, Spectral) that resulted of 0.96 ( $>0.60$ higher risk of sepsis). The patient began empirical support antibiotic therapy with teicoplanin, meropenem and fluconazole.

Blood cultures sent to the laboratory at the day of admission were negative until the 3rd day of aerobic incubation. Subsequently, both the blood cultures and the liquid peritoneal drainage showed a growth of Gram-negative bacilli difficult to identify after $48 \mathrm{~h}$ of aerobical incubation. Such bacteria were identified as E. meningosepticum and antibiogram showed a characteristic profile of multidrug resistance to most of the antibiotics useful against Gram-negatives but susceptibility to those targeting Gram-positives (vancomycin, ciprofloxacin, etc.). Empirical antibiotic therapy was replaced by a specific therapy with the following antibiotics: vancomycin, trimethoprim/sulfamethoxazole, ciprofloxacin and levofloxacin (times, dosages and adminis- tration protocols were not available). During the antibiotic treatment the endotoxin dosage was halved with 0.55 values (medium risk), showing that the patient responded clinically to therapy. Blood cultures performed after antibiotic therapy (about 2 weeks) were sterile, confirming the elimination of E. meningosepticum infection.

Unfortunately, during hospitalization in Intensive Care and, in particular, in the weeks following the isolation and subsequent the eradication of E. meningosepticum, the patient's condition worsened. Clinical complications, occurred as consequence of a partial gastrectomy, with removal of peritoneal tissue, pleural effusion, congestive acute kidney failure and presence of secondary infections in different body districts by Acinetobacter baumannii (MDR) and Staphylococcus aureus species The clinical conditions, inevitably, increasingly worsened, until the onset of sepsis from Acinetobacter baumannii (MDR) -evolved in a short period in septic shock and cardiac arrest, with decease of the patient.

\section{Materials and methods}

Two sets of blood cultures were collected from the patient A.A. in different dates: firstly during the hospitalization in Intensive Care and secondly after six days, due to the presence of persistent fever $\left(38-39^{\circ} \mathrm{C}\right)$. Different microbiological samples were collected and sent to the laboratory during the hospital stay: peritoneal fluid drainage, tracheal aspirate, central venous catheter culture etc. Blood was cultured in 1 BACTEC Plus/F Aerobic, 1 BACTEC Plus/F anaerobes and 1-BACTEC Mycosis IC/F, while fluid drainages were cultured in 1 BACTEC Plus PED; the samples were incubated in BD BACTEC тм 9000System. The different samples were processed using the main microbiological culture media (BD) available, according to our internal procedures. The positive bottles were seeded on Chocolate, Blood, Mac-Conkey, Mannitol Salt, Sabouraud agar and incubated at $37^{\circ} \mathrm{C}$ overnight. The colonies were subjected to Gram-staining, while identification and antibiotic susceptibility testing were achieved preparing an inoculum of 0.6 and $0.8 \mathrm{McF}$ respectively, and using the card GN and AST-201 (and AST632), the system Vitek2, (bioMérieux, Marcy-l'Étoile, France). Other biochemical tests used for identification were: catalase test, oxidase test, and in particular the indole test stick (Liofilchem, Roseto degli Abruzzi, Italy) which shows the tryptophanase activity of the enzyme which degrades the aminoacid tryptophan into pyruvic acid, ammonium and indole, the latter highlighted by an aldeid indicator that develops an intense green colour.

The identification was confirmed by MALDI-TOF method, VitekMS, bioMérieux: 1-2 fresh isolated colonies, grown in plate according to routine protocols ( 48 hours), were placed with the tip of a micropipette at the centre of a spot of a target plate disposable polycarbonate with the addition of 1 $\mu \mathrm{L}$ of matrix made of organic materials ( $\alpha$-cyan-4-hydroxycinnamic acid) that can provide a source of protons necessary for the ionization of the sample. Further antibiotic susceptibility tests were performed by E-test (bioMérieux), 
preparing an inoculum $0.8-1 \mathrm{McF}$ in physiological solution, and using Chocolate agar plates. A phenotype analysis aimed to confirm the expression of metallo-beta-lactamases (MBL) on the strain of E. meningosepticum through a synergy test was also performed (ROSCO Diagnostics, Taastrup, Denmark). A difference $\geq 5 \mathrm{~mm}$ between the Meropenem inhibition zone -of and the one of Meropenem + dipicolinic acid indicates the production of a MBL.

\section{Results}

Blood cultures from the bottle in aerobic condition resulted positive in both sets, after 3 days of incubation at $37^{\circ} \mathrm{C}$, while after only $48 \mathrm{~h}$ the bottle was positive PED Plus in which the liquid peritoneal drainage had been inoculated. The bacterial culture of the different biological samples (blood cultures, drainage liquid, etc.), showed stunted growth to $24 \mathrm{~h}$ and to $48 \mathrm{~h}$ more marked only on Chocolate agar and Blood agar. The colonies on Chocolate agar showed a distinctive grey/whitish-opaque (due to protease and gelatinase action), without yellow pigmentation, and with mucosal aspect, translucent, reflective surface and with sharp margins, with a 1-2 mm diameter. On Blood agar such characteristics appeared more marked and evident, and, in addition, it was noted a tight beta-haemolysis (Figure 1). After Gram-staining, optical microscope observation (1000× immersion), showed the presence of small Gram-negative bacilli, smoothing and intensely coloured (Figure 2). Although is the presence of a Gram-negative, the bacterium didn't grow on Mac Conkey agar, even after $72 \mathrm{~h}$ of incubation.
The colony was then analysed for the biochemical identification of Vitek2 system using the card GN and was identified as E. meningosepticum with probability of $99 \%$ and excellent reliability. The catalase test and oxidase were, as expected, were both positive.

As described in literature, this species may be confused by automatic systems with that of Aeromonas genus, and, moreover, it is important to place a differential diagnosis with Burkholderia cepacia species, also a Gram-negative non-fermenter bacillus, with no growth of Mac Conkey agar in less than 3 days (slow growth) and with a profile of susceptibility to similar antibiotics (colistin-resistant, vancomycin-susceptible). To distinguish between the two genres were backed indole test which must be clearly negative for Aeromonas and Burkholderia, contrarily, decidedly positive for Elizabethkingia. The final confirmation of the identification that the bacterium belonged to E. meningosepticum species has occurred from the analysis of MALDITOF mass spectrum of the colony, compared with the reference spectra in the database, with the confidence score of the spectrum obtained of $96 \%$ (Figure 3 ). In addition, $E$. meningosepticum colonies were sent to the Microbiology Laboratory of the Hospital San Filippo Neri in Rome (Italy) for further confirmation of species identification.

The antibiogram performed with the card AST-201 on Vitek2, showed that E. meningosepticum had a MDR profile. In particular, the bacterium was resistant to most antibiotics useful in the case of Gram-negative bacteria, including colistin (MIC $\geq 16 \mathrm{mg} / \mathrm{L}$ ); it was instead susceptible to molecules normally effective against Gram-positive bacteria, such as vancomycin, trimethoprim-sulfamethoxazole, ciprofloxacin, levofloxacin (Table 1). The susceptibility profile was also

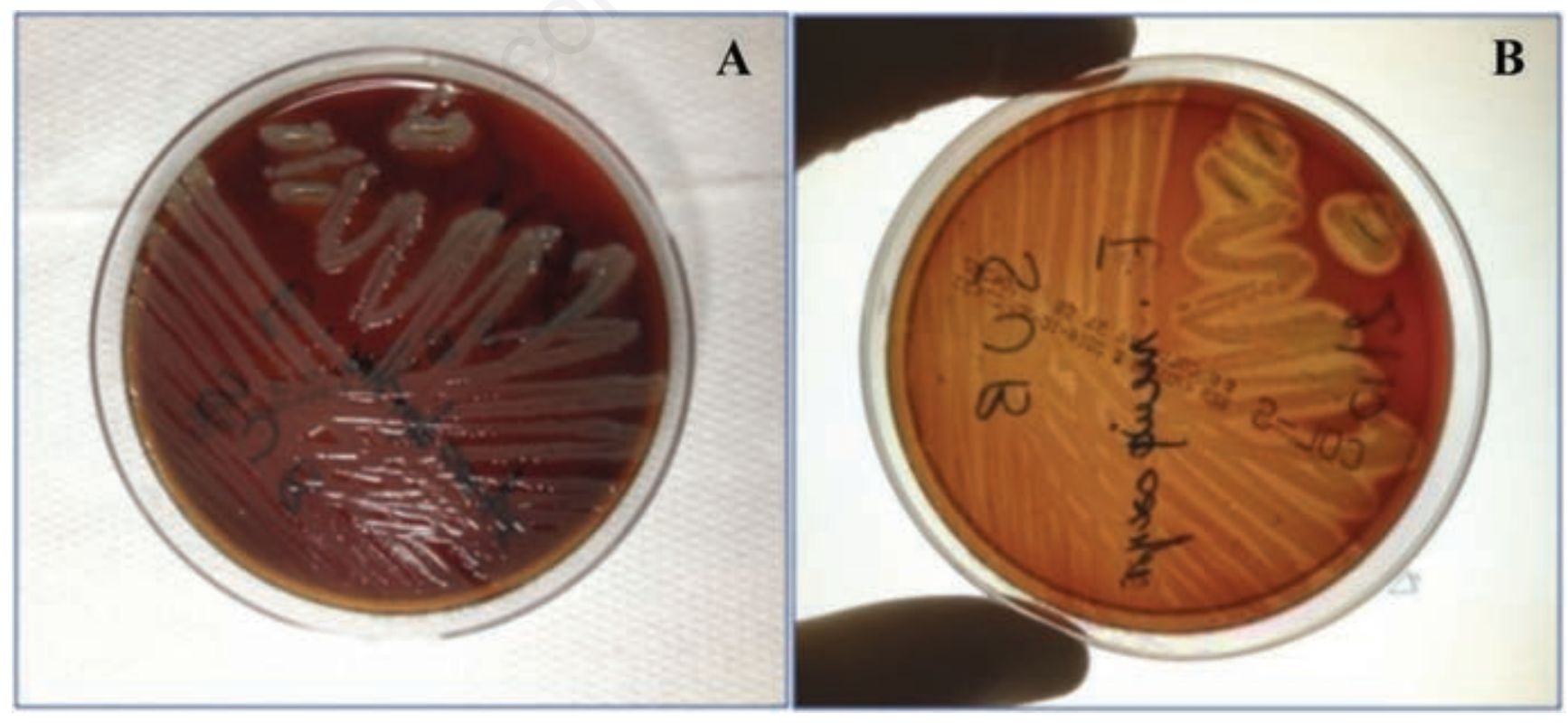

Figure 1. A) E. meningoseptimum colonies on Chocolate agar showing a characteristic grey/whitish-opaque colour (protease and gelatinase action), no yellow pigmentation, with mucose aspect, translucent, reflective surface, with sharp edges, and 1-2 mm diameter. B) $E$. meningoseptimum colonies on blood agar show the same macroscopic characteristics found on chocolate agar but more evident; it is noted a tight beta-haemolysis of the colonies. 
confirmed using the card AST-202 (AST-201) and the card AST-P632, the latter used particularly to test the vancomycin susceptibility (MIC $=8 \mathrm{mg} / \mathrm{L}$ ), levofloxacin $(\mathrm{MIC}=0.5 \mathrm{mg} / \mathrm{L}$ ), and other antibiotics active against Gram-positives (Table 1). The MIC of colistin and meropenem was also determined by E-test, confirming both the resistance to colistin (MIC $\geq 16$ $\mathrm{mg} / \mathrm{L}$ ) and carbapenems (e.g. Meropenem (MIC $\geq 32 \mathrm{mg} / \mathrm{L}$ ), and the susceptibility to both ciprofloxacin (MIC $=0.5 \mathrm{mg} / \mathrm{L}$ ) and trimethoprim-sulfamethoxazole (MIC $\leq 20 \mathrm{mg} / \mathrm{L}$ ), (Table 1). The MICs were interpreted using CLSI recommendations (used by most of the Authors for E. meningosepticum), often overlapping with EUCAST. The breakpoint used for the interpretation of MIC for vancomycin in Staphylococcus aureus was, instead, EUCAST. As shown in Table 1, a vancomycin $\mathrm{MIC}=4 \mathrm{mg} / \mathrm{L}$ in an E-test, was lower than the one obtained with the card AST-P632 (MIC=8 mg/L). This caused some embarrassment in assigning category (SIR), as both MIC values are interpreted as resistant by EUCAST, while intermediate by CLSI. As many Authors follow the recommendations CLSI, and considering that the vancomycin is often used for initial treatment in infections by E. meningosepticum, it was decided an intermediate category for the vancomycin result of our bacterial strain (Table 1). In addition, E. meningosepticum is naturally resistant to beta-lactams, including carbapenems, due to a beta-lactamase overproduction. To confirm the carbapenem resistance data, a phenotypical synergy test was performed, and it resulted positive.

\section{Discussion}

Most cases of Elizabethkingia meningosepticum infections are acquired in the hospital and usually involve immunosuppressed patients, polytrauma and cases of meningitis in infants. Although very rare, there was, during the last decade, an increase of nosocomial infections caused by this pathogen, that can be fatal if not early diagnosed and treated. Our case is probably one of the first documented in Italy. It describes a sepsis caused by E. meningosepticum in patients already seriously compromised by a severe neurological disorder. It is possible that the contact between the pathogen and the patient took place during the hospital stay, but no other cases of infection due to E. meningosepticum

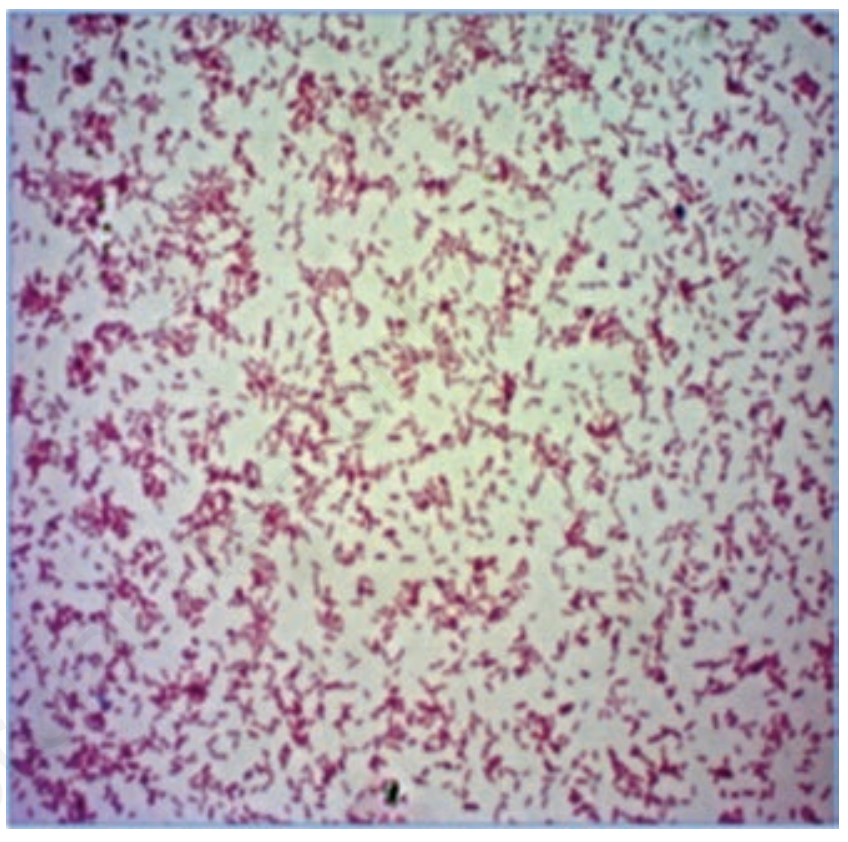

Figure 2. Optical microscope observation (1000× immersion), show the presence of small gram-negative bacilli.

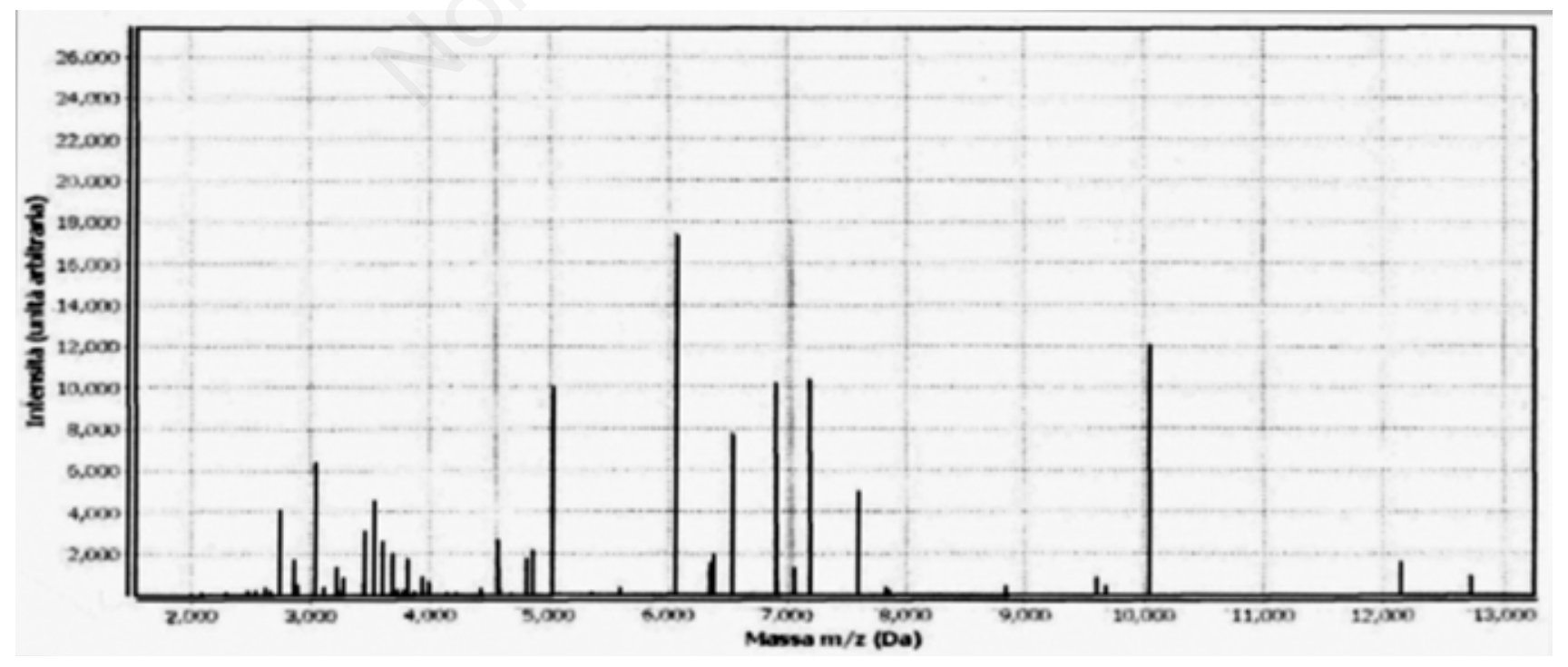

Figure 3. MALDI-TOF spectrogram of E. meningoseptimum colonies on chocolate agar: excellent reliability $96 \%$. 
Table 1. Susceptibility test results obtained by Vitek 2 and E-test.

\begin{tabular}{|c|c|c|c|c|c|c|c|}
\hline Antibiotic AST 201 & $\begin{array}{c}\text { MIC } \\
(\mathrm{mg} / \mathrm{L})\end{array}$ & SIR & Antibiotics ASTP632 & $\begin{array}{c}\mathrm{MIC} \\
(\mathrm{mg} / \mathrm{L})\end{array}$ & SIR & E-test (MIC mg/L) & SIR \\
\hline Amikacin & $\geq 64$ & $\mathrm{R}$ & Benzilpennicillin & $\geq 0.5$ & $\mathrm{R}$ & Amikacin (256) & $\mathrm{R}$ \\
\hline Amoxicillin/Ac. Clav. & $\geq 32$ & $\mathrm{R}$ & Oxacillin & $\geq 4$ & $\mathrm{R}$ & - & - \\
\hline Ampicillin & $\geq 32$ & $\mathrm{R}$ & Levofloxacin & 0.5 & $\mathrm{~S}$ & Levofloxacin (0.38) & $\mathrm{S}$ \\
\hline Cefepime & $\geq 64$ & $\mathrm{R}$ & Eritromicin & $\geq 8$ & $\mathrm{R}$ & - & - \\
\hline Cefotaxime & $\geq 64$ & $\mathrm{R}$ & Clindamicin & 0.5 & I & - & - \\
\hline Ceftazidime & $\geq 64$ & $\mathrm{R}$ & Linezolid & $\geq 8$ & $\mathrm{R}$ & - & - \\
\hline Ciprofloxacin & 0.5 & $\mathrm{~S}$ & Daptomicin & $\geq 8$ & $\mathrm{R}$ & Ciprofloxacin (0.5) & $\mathrm{S}$ \\
\hline Colistin & $\geq 16$ & $\mathrm{R}$ & Teicoplanin & $\geq 32$ & $\mathrm{R}$ & Colistin (256) & $\mathrm{R}$ \\
\hline Ertapenem & $\geq 8$ & $\mathrm{R}$ & Vancomicin & 8 & $\mathrm{I} / \mathrm{R}$ & Vancomicin (4) & I \\
\hline Gentamicin & 8 & $\mathrm{R}$ & Gentamicin & 2 & I & Gentamicin (8) & $\mathrm{R}$ \\
\hline Meropenem & $\geq 16$ & $\mathrm{R}$ & Tetraciclin & $\geq 16$ & $\mathrm{R}$ & Meropenem (32) & $\mathrm{R}$ \\
\hline Nitrofurantoin & $\geq 512$ & $\mathrm{R}$ & Fusidic Acid & 8 & $\mathrm{R}$ & - & - \\
\hline Tigeciclin & 2 & $\mathrm{R}$ & Rifampicin & $\geq 4$ & $\mathrm{R}$ & - & - \\
\hline Trimetropim/Sulfam. & $\leq 20$ & $\mathrm{~S}$ & Trimetropim/Sulfam. & $\leq 20$ & $\mathrm{~S}$ & Trimetropim/Sulfam.(0.12) & $\mathrm{S}$ \\
\hline
\end{tabular}

occurred, and no environmental monitoring have been conducted to identify the source of infection. It cannot have been excluded that the patient might have acquired the pathogen within home care, given his condition.

As described in literature, it is very difficult to reach an E. meningosepticum identification from blood cultures. The macroscopic and microscopic features of the colonies, the growth mode on different culture media along with identification with different systems (Vitek2 and MALDI-TOF) have recognized the isolate as E. meningosepticum relatively quickly. Nevertheless, biochemical identification performed with automated systems should always be confirmed adding other tests (such as the indole), to allow a reliable diagnosis able to distinguish from bacteria belonging to the genus Aeromonas and Burkholderia.

E. meningosepticum has a specific and atypical antibiotic profile. For this reason, it is evident that define an effective antibiotic therapy is very difficult. Our E. meningosepticum strain was susceptible to the following antibiotics: ciprofloxacin, levofloxacin, trimethoprim/sulfamethoxazole (Table 1). Therefore it was suggested to the Department of Intensive Care the use of such molecules, including vancomycin, that, albeit the intermediate category obtained (CLSI), is often recommended for the initial treatment of infections caused by E. meningosepticum. As described by other Authors, a monotherapy was discouraged, and the use of two or more antibiotics in combination was proposed. In the present clinical case, this approach resulted effective, and led to the resolution of E. meningosepticum sepsis.

Moreover, it was difficult to establish a possible correlation between the antibiotic therapy used for E. meningosepticum, and the infection by A. baumannii (MDR) (usually colistinsusceptible), subsequently affecting the patient.

\section{Conclusions}

In conclusion, our work documents that for intensive care patients, considering the possibility of unusual Gramnegative bacilli as opportunistic pathogens responsible of infection, can lead to an early change in the empirical antibiotic therapy to a more targeted one, with a significant reduction in morbidity and mortality. Moreover, given the increased incidence of infections with E. meningosepticum, would be desirable for the future to plan more studies aiming at its genotypic and phenotypic characterization thus enabling earlier diagnosis and better overall management.

\section{References}

1.Bomb K, Arora A, Trehan N. Endocarditis due to Chryseobacterium meningosepticum. Indian $\mathrm{J}$ Med Microbiol 2007;25:161-2.

2. Ceyhan M, Celik M. Elizabethkingia meningosepticum (Chryseobacterium meningosepticum) infections in children. Int J Pediatr 2011;2011:215237.

3. Dias M, Fernandes A, Furtado Z. Case series: Elizabethkingia meningosepticum. J Clin Diagn Res 2012;6:1550-1.

4. Fraser SL, Jorgensen JH. Reaprisal of antimicrobial susceptibilities of Chryseobacterium and Flavobacterium species and method for reliable susceptibility testing. Antimicrob Agents Chemother 1997;41:2738-41.

5. Gokul BN, Chandramukhi A, Ravikumar R, Aroor S. Flavobacterium meningosepticum meningitis in a neonate. Indian J Pediatr 1989;56:524-7. 
6. Hsu MS, Liao CH, Huang YT, et al. Clinical features, antimicrobial susceptibilities, and outcomes of Elizabethkingia meningoseptica (Chryseobacterium meningosepticum) bacteremia at a medical centre in Taiwan, 1999-2006. Eur J Clin Microbiol Infect Dis 2011;30:1271-8.

7. Issack MI, Neetoo Y. An outbreak of Elizabethkingia meningoseptica neonatal meningitis in Mauritius. J Infect Dev Ctries 2011;5:834-9.

8. Kim KK, Kim MK, Lim JH, et al. Transfer of Chryseobacterium meningosepticum and Chryseobacterium miricola to Elizabethkingia gen. nov. as Elizabethkingia meningoseptica comb. nov. and Elizabethkingia miricola comb. nov. Int J Syst Evol Microbiol 2005;55:1287-93.

9. King EO. Studies on a group of previously unclassified bacteria associated with meningitis in infants. Am J Clin Pathol 1959;31:241-7.

10. Kirby JT, Sader HS, Walsh TR, Jones RN. Antimicrobial susceptibility and epidemiology of a worldwide collection of Chryseobacterium spp: report from the SENTRY antimicrobial surveillance program (1997-2001). J Clin Microbiol 2004;42:445-8.
11. Lin PY, Chu C, Su LH, et al. Clinical and microbiological analysis of bloodstream infections caused by Chryseobacterium meningosepticum in non neonatal patients. J Clin Microbiol 2004;42:3353-5.

12. Nisel O, Murat A, Neval A, et al. Community acquired meningitis and sepsis caused by Cryseobacterium meningosepticum in a patient diagnosed with thalassemia major. J Clin Microbiol 2006;44:3037.

13. Padmaja $\mathrm{P}$, Verghese $\mathrm{S}$, Bhirmanandham $\mathrm{CV}$, et al. Chryseobacterium meningosepticum: an uncommon pathogen causing adult bacterial meningitis. Indian $\mathrm{J}$ Pathol Microbiol 2006;49:293-5.

14. Sarma S, Kumar N, Jha A, et al. Elizabethkingia meningosepticum: an emerging cause of septicaemia in critically III patients. J Lab Physicians 2011;3:62-3.

15. Thong ML, Pudthucheary SD, Lee EL. Flavobacterium meningosepticum infection: an epidemiological study in a newborn nursery. J Clinpathol 1981;34:429-33.

16. Weaver KN, Jones RC, Albright R, et al. Acute emergence of Elizabethkingia meningoseptica infection among mechanically ventilated patients in a long term acute care facility. Infect Control Hosp Epidemiol 2010;31:54-8. 